\title{
UNA PROPUESTA PARA ESTUDIAR EL PAISAJE A ESCALA REGIONAL EN EL PERÚ
}

\author{
A PROPOSAL TO STUDY LANDSCAPE AT REGIONAL SCALE IN PERU
}

\author{
${ }^{1}$ Victor Peña Guillén
}

\section{Resumen}

En el presente documento se sugiere, a manera de ensayo y recopilación bibliográfica, una perspectiva para el estudio del paisaje a escala regional con fines de evaluación y planeamiento espacial. El estudio busca mostrar la importancia de representar y analizar el patrón de los componentes espaciales del paisaje (abiótico, biótico y cultural) desde el marco teórico de la Ecología del Paisaje, resaltando la importancia del estudio para comprender el funcionamiento de los ecosistemas, sobretodo en casos donde existe presión de cambio de uso del suelo hacia actividades humanas. Se muestran dos ejemplos de cambio en el paisaje que se caracterizan tanto por su escala regional de influencia, como por la rapidez con la que sucede el cambio: la urbanización de los valles donde se asienta el área metropolitana de Lima, y la explotación minera a tajo abierto en el área de operaciones del complejo Yanacocha. El carácter del ensayo busca ir mas allá de la visión tradicional de achacar a las actividades humanas (seguramente con justificada razón), todos los impactos negativos en el paisaje y los problemas ecológicos y perceptuales subsecuentes. La sugerencia se refiere a una visión alternativa del paisaje, donde se analiza una escala física y perceptual establecida por los usos de los recursos y la percepción de parte del ser humano. El tamaño de la superficie de esta escala de paisaje propuesta permite entender mejor las interrelaciones entre el paisaje y el uso y percepción humana, considerándose apropiado tomar como referencia conceptual a la Ecología del Paisaje.

Palabras clave: Planeamiento del Paisaje Regional, Ecología del Paisaje, Escala del Paisaje, Cambio del uso del suelo.

\begin{abstract}
This study uses essay narrative in order to suggest and argues how important landscape study is from a regional scale approach. Practical uses of the study on this regional scale are referred to landscape assessment and planning. Landscape study focuses first on the ecosystem configuration and its perception as a whole spatial model. Theoretical reference to help describe and understand landscape described in the above terms is provided by Landscape Ecology. Two case studies are selected to better explain the importance of regional landscape study: first the urbanization process in Metropolitan City of Lima, and second, landscape disturbance caused by open pit mining operations in Yanacocha mine. Both examples have common issues: regional scale and rapid land use change. This essay is focused not in describe topics relative to negative impacts of the described cases, but to provide a comprehensive view to manage such rapid changes in regional landscape character and the abiotic, biotic and cultural processes related to the changes; Landscape Ecology helps to address the problems described and are easily linked with complementary themes as transdisciplinarity approach, which hence support informed decisions for public and private stakeholders in landscape and land use policies.
\end{abstract}

Key words: Landscape Regional Planning, Landscape Ecology, Landscape Scale, Land Use Change.

\section{Introducción}

El significado y el uso del concepto paisaje en el país es generalmente relacionado a la práctica y verbo del paisajismo, entendido este como la actividad de crear espacios verdes en un angosto rango de tamaños que va desde el jardín hasta el parque urbano. En el presente texto se sugiere utilizar el término del paisaje, y su enfoque de estudio, de tal manera que se extienda la escala y aplicación conceptual del termino hacia superficies de un alcance geográfico mayor, que incluyan el tamaño de las regiones urbanas, los valles agrícolas y las cuencas hidrográficas. A continuación presentare una definición de paisaje que considero adecuada para enmarcar el concepto y significado que se propone dar al paisaje en el presente documento. Según Antrop (2005), el paisaje no solo se refiere al complejo fenómeno físico que puede ser descrito y analizado utilizando métodos objetivos y científicos; Antrop extiende el concepto (citando a Cosgrove y Daniels 1988; Lowenthal 1975; 1985), y de acuerdo a esta ampliación del término, el paisaje también se refiere a la observación y experiencia subjetiva, lo cual 
a su vez le brinda un significado perceptivo, estético, artístico y existencial.

Este incremento de la amplitud de la escala espacial y en el significado del paisaje requiere, para su estudio científico y su diseño creativo, un marco teórico y herramientas específicas para el análisis del espacio con las que se pueda representar gráficamente y modelar la estructura física, y el conjunto de los procesos naturales y culturales que están contenidos en ese espacio, teniendo como objetivo practico del estudio, en primer lugar, la evaluación de la condición ecológica y el carácter del paisaje en la región, y también el planeamiento del cambio futuro. Se entiende que la complejidad de la estructura física a grandes escalas, y los procesos abióticos, bióticos y culturales que intervienen en ella, requieren un enfoque interdisciplinario; según Tress y Tress (2005), el concepto interdisciplinario se refiere a la integración operativa de diferentes disciplinas, no relacionadas entre si, de manera de forzar su interrelación cruzando sus propios límites teóricos y metodológicos, en la búsqueda de dar solución a un objetivo común. Al extender la escala de estudio hacia espacios de gran superficie se hace necesaria una perspectiva estratégica para la gestión del espacio y el manejo de los componentes sociales, económicos y ambientales. Las decisiones de cambio en el paisaje a gran escala implican asumir un riesgo mayor de equivocación en la localización de actividades y recursos, teniendo en cuenta la amplitud espacial, los recursos económicos y la población que serían afectados negativamente. Esta mayor superficie del paisaje regional está relacionada directamente con una mayor complejidad del estudio, evaluación y planeamiento, y supone también aceptar una mayor dedicación de tiempo y recursos en los estudios, los cuales deberían orientarse hacia la integración eficaz de las disciplinas profesionales que estudian el paisaje, ya que su mencionada complejidad y su naturaleza sistémica así lo requiere. La visión integradora, tanto del equipo de estudio como del enfoque de evaluación y planeamiento, contrasta con la practica actual de trabajo en los estudios ambientales que la normatividad exige, según la cual la integración se hace efectiva principalmente en las etapas finales del trabajo multidisciplinario, y de acuerdo a los requerimientos que las normas públicas o el promotor o cliente privado requieren.

La narrativa a seguir en la exposición de la presente propuesta acerca del estudio del paisaje sigue un orden en el cual se exponen dos casos fundamentales que explican la dinámica de cambio actual que ocurre en el territorio peruano: el primero de ellos es el crecimiento urbano en ciudades grandes y medianas, sobretodo en capitales de región y de provincia, y el segundo caso es la importante modificación del paisaje regional causado por la gran minería, localizada en zonas rurales altoandinas.

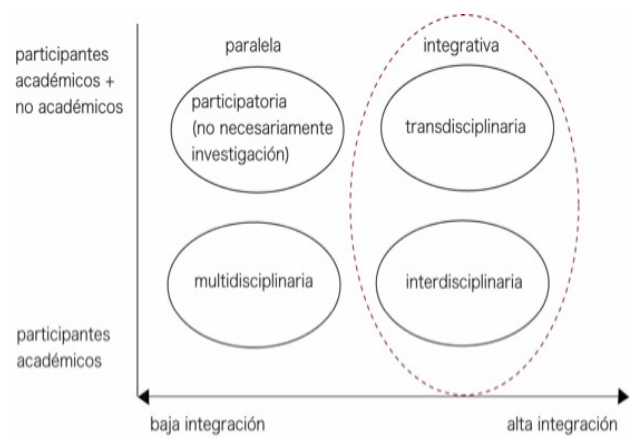

Figura 1. Grado de integración de los participantes en el estudio del paisaje. Elaboracion propia tomado de Tress, Tress y Fry (2004).

Ambos casos tienen denominadores comunes tales como la rapidez con la que los cambios en el uso y cobertura del suelo ocurren, y la escala regional de influencia económica, ambiental y social; además de que, tanto la ciudad de Lima como el complejo minero Yanacocha, ha llegado a constituir de manera creciente en los motores del desarrollo económico nacional. Hecha esta primera descripción de la evidencia del cambio en el paisaje, en los dos casos específicos mencionados, sería conveniente detenerse en establecer un hecho de constante ocurrencia: la estructura física del espacio que nos rodea siempre está cambiando, ya sea tanto como consecuencia de fenómenos naturales como los terremotos, sequias, inundaciones, la erosión o los deslizamientos de tierras, así como también por causa de la actividades humanas generadas por la demanda y uso de los recursos naturales y espacio físico. Respecto a esto último se viene observando en los últimos años que hay un incremento en el requerimiento de superficies y recursos naturales para construir y mantener operativas carreteras, ciudades, minas, embalses de agua, etc. Este cambio constante en el paisaje implica la ocurrencia y cambio de procesos complejos muchas veces subyacentes (hidrológicos, físico-químicos, culturales, etc.), que se reflejan en nuevas formas del paisaje, cuya percepción contiene en general elementos artificiales descontextualizados y contrastantes del carácter del espacio que ocupan. La velocidad del cambio en el paisaje es en todo caso acelerado por actividades y decisiones de una sociedad a su vez cambiante rápidamente en ingresos económicos, tamaño poblacional y valores sociales. Al haber descrito, de manera muy general, las ocurrencias actuales en la dinámica del cambio del paisaje en el Perú, y luego de sugerir dos ejemplos donde esta dinámica se desarrolla a gran velocidad, nos quedaría proponer algunas ventajas que aparecen como resultado de la lógica funcional del pensamiento descrito. Esta lógica partió del reconocimiento y la importancia del problema, la propuesta de solución a través del estudio del paisaje total y la proyección metodológica acompaña y posibles alcances en el cambio de la forma de observar el cambio y su gestión. Una de las ventajas 
de la presente propuesta de estudio del paisaje regional, es el reconocimiento de la conveniencia de orientar espacialmente y hacer compatible la demanda de recursos y la inversión económica, con el cuidado del carácter y función del paisaje, en aquellos sectores de cambio físico del paisaje, manteniéndolo atractivo y consecuente con los usos y preferencias de la población y los grupos de interés involucrados. Otra ventaja de un estudio territorial utilizando el concepto de paisaje, en una escala regional, y el aporte hacia una eficiente ocupación y uso del territorio, en la condiciones actuales de cambio acelerado, consiste en proveer una visión del conjunto: tanto de la estructura física (su esqueleto), como de los procesos vitales (su metabolismo), todo lo cual influye en el cambio. La sugerencia en general se basa en el entendido que observando cuidadosamente y manteniendo saludable las funciones naturales, se construye la base física franca y sincera que se refleja en un paisaje que ofrece una apreciación y percepción positivas desde la población y los actores públicos y privados interesados en el bienestar particular y público. A continuación sugeriremos, y justificaremos en mayor profundidad, la conveniencia de utilizar el concepto de paisaje en el estudio integral del territorio a escala regional tanto para su evaluación $\mathrm{y}$ entendimiento como para su planeamiento futuro. El lineamiento metodológico que se expondrá, estima tenga a bien, tomar en cuenta e informar conceptos que se consideran importantes en la decisiones planificadas de cambio en el paisaje. Se considera que las sugerencias así expuestas puedan servir para transmitir, a los actores públicos y privados que intervienen en las decisiones que influyen en el cambio del paisaje, inquietudes acerca de la evidencia actual de cambio acelerado, en las regiones urbanas y algunos sectores altoandinos relacionados con la actividad minera. Asimismo el presente texto busca advertir acerca de la importancia de conocer y relacionar en un conjunto los procesos y estructura de un área de estudio regional, de modo que la decisión resultante de la negociación entre ellos, se enlace directamente al entorno espacial, y lo tome en cuenta en las decisiones en la correspondiente escala del paisaje, y en el nivel adecuado de proposición e implementación de políticas públicas o corporativas. Un alcance importante que requiere aclaración explicita es que el concepto del paisaje y sus herramientas de evaluación y planeamiento forman parte del desarrollo urbano o el desarrollo rural, es decir el planeamiento espacial está directamente relacionado con la implementación y realización de los emprendimientos e inversión pública y privada en beneficio de la sociedad. El paisaje, definido por el Convenio Europeo del Paisaje (2000), es "el territorio, tal como lo percibe la población, cuyo carácter es el resultado de la acción y/o interacción de factores naturales y humanos"; este se considera como un concepto que ayuda a relacionar y entender el sistema integral de los componentes ambientales, sociales y económicos del territorio; se considera asimismo que su estudio no es un fin en sí mismo, sino que más bien colabora en la búsqueda del fin mayor del bienestar de la sociedad.

\section{Materiales y métodos}

El paisaje está compuesto en conjunto por el territorio, es decir sus características y elementos físicos dentro de un espacio, y por el componente perceptivo del observador humano; la percepción convierte al territorio en paisaje y le da un valor subjetivo, en conjunto con sus componentes abióticos, bióticos y culturales; además le confiere un carácter distintivo respecto a otro paisaje; en general las características individuales o personalidad de un paisaje no lo hacen mejor ni peor al momento de comparar subjetivamente diferentes paisajes; la importancia de conocer el carácter es tener el conocimiento necesario para decidir cuál es el uso adecuado y sostenible según el criterio elegido por los agentes de toma de decisión y las recomendaciones presentadas por los especialistas y científicos. En el Perú la geología y el clima han producido una enorme cantidad de paisajes con un carácter particular; existen paisajes costeros áridos, desembocaduras de ríos con humedales, bosques ribereños, centros urbanos, valles bajo riego, laderas con vegetación temporal, tierras agrícolas en pendientes escarpadas, bofedales altoandinos, lagunas, etc. Esta variedad de paisajes se ha incrementado con la intervención humana que en el espacio de franja costera, y los andes centrales sudamericanos, ha sido especialmente generosa en la creación de cultura y en la modificación y adaptación del paisaje a usos de explotación primaria en armonía y dependiente con las condiciones naturales. Teniendo en cuenta la importancia económica del aprovechamiento de los recursos naturales, el patrón inicial de uso agrícola y comercial de una primera etapa de ocupación y uso del suelo dio lugar, vigente desde el descubrimiento de la agricultura en los andes, dio lugar a un cambio tecnológico ocurrido hace más de un siglo, que condujo a la aparición de un modelo extractivo de minerales e industrial de carácter generalmente primario, el cual acompañado de la construcción de infraestructura vial. Esta siguiente era de aprovechamiento de los recursos naturales, a una mayor escala espacial y volumen productivo, dio como resultado una mayor movilización poblacional e inmigración hacia los centros urbanos de la costa y sierra, y en menor medida hacia asentamientos rurales en la selva, por ejemplo a partir de la construcción de la carretera marginal de la selva. El presente texto busca describir primero y entender después el modo como es que esta segunda etapa histórica de aprovechamiento de los recursos y espacios naturales está influyendo en la actualidad en la estructura y funcionamiento en los paisajes de las regiones y cuencas. La observación y representación cartográfica del paisaje muestra evidencia clara de un cambio acelerado en el uso del suelo y en el carácter de los paisajes urbanos en ciudades grandes y medianas del Perú, y lo mismo viene ocurriendo en zonas de actividad minera, de gran escala, sobretodo en operaciones de tajo abierto en regiones altoandinas.

El marco legal de las normas de planificación en el Perú, aplicado en aquellos lugares donde existe una planificación del uso del suelo, orienta las decisiones de planificación y desarrollo, por ejemplo el Modelo de Desarrollo Urbano 
para Pisco (2012) regula las características y zonifica el uso del suelo de acuerdo a lineamientos consideradas y establecidas previamente en las normas y políticas específicamente el Reglamento de Acondicionamiento Territorial y Desarrollo Urbano (DS 004-2011-Vivienda); en aquellas zonas con normativa de ocupación espacial, como la del ejemplo anterior, se indican aquellas actividades permitidas o prohibidas, tamaño de los lotes destinados a hoteles o casas, ubicación de los comercios, altura de construcción, entre otros lineamientos basados en la planificación urbana que busca proveer las condiciones y equipamiento para el desarrollo de las actividades humanas. Según Forman (2008), el planeamiento urbano busca promover el crecimiento inteligente; el enfoque del paisaje y su estudio lo complementa y lo orienta hacia un planeamiento donde se resalta las condiciones naturales y perceptivas del lugar, que permiten sostener, con el menor costo económico, social y ambiental, a las actividades humanas.

El estudio y análisis de las alternativas eventuales, para el planeamiento del uso del suelo, basadas en las condiciones y aptitud del territorio, no se encuentran previstas explícitamente en las leyes, reglamentos y códigos peruanos. Para planificar y establecer normas que dirijan el planeamiento a partir de una análisis transparente, holístico y científico del territorio (pues se entiende que la ciencia se aproxima más a la verdad), se propone el concepto de paisaje como fundamento de un estudio participativo o transdisciplinario, donde concurran tanto los técnicos como los grupos de interés, para realizar el diagnóstico y luego el planeamiento espacial. Una evaluación imparcial del funcionamiento del paisaje se facilita al existir un marco de planificación y normas explícitas que se apliquen al uso del suelo y su percepción. Entonces como primer paso se hace necesario que el concepto, y la actividad de evaluación del paisaje, se explicite mejor en la normatividad existente, y además trascienda a las normas puesto que las leyes pueden cambiar de la noche a la mañana mientras que los sistemas naturales se mantienen bajo leyes más durables. La apreciación del carácter del paisaje resulta fundamental para su planeamiento y gestión. El carácter del paisaje es el resultado de la acción e interacción de los factores naturales y humanos (Convenio Europeo del Paisaje 2000). La dinámica de cambio en los sistemas naturales toman un horizonte tiempo extenso y su desarrollo es a largo plazo. El cambio en el paisaje producto de las actividades humanas y la legislación ocurre en plazos más cortos; en el Perú estos cambios son percibidos con mayor facilidad en los valles agrícolas, y en sectores puntuales de las zonas rurales altoandinas.

Como regla general, es óptimo y útil considerar primero que el cambio produce impactos sobre el paisaje y que, cuando no resultara posible evitarlos, es inteligente estudiar y aplicar estrategias de reducción, remediación, compensación y/o mejora, estudiando y eligiendo entre diferentes alternativas, lo cual debería estudiarse desde la etapa de diseño del proyecto. La anterior recomendación se puede implementar mejor a partir de una certera representación del paisaje actual (que depende de los objetivos de estudio la escala espacial y los recursos comprometidos). Una representación de la estructura y el metabolismo subyacente y que mantiene dicha estructura del paisaje regional permite responder la siguiente pregunta: ¿cómo opera el paisaje? Consideramos clave la respuesta pues permitirá comprender y evaluar la condición actual del paisaje y, a partir de esa comprensión y el conocimiento adquirido, proponer alternativas de cambio que complementen las normas de uso del suelo, e introduzcan la influencia espacial que tiene el sistema territorial abierto a las leyes de la naturaleza y la sociedad. El resultado que idealmente se espera de esta lógica de estudio del paisaje localizar espacios de alto y bajo valor ecológico donde se adapten las correspondientes actividades humanas brindando un sólido futuro al sistema territorial de la región.

A modo de comentario y alcance del estudio del paisaje aplicado actualmente, cabe mencionar que la actual normativa peruana para la realización de los Estudios de Impacto Ambiental - EIA (2008), requiere la descripción o representación, del estado actual del área de actuación, previa a la ejecución de un proyecto. Los estudios exigidos comprenden la descripción detallada de los atributos o características socio ambientales del área de emplazamiento de un proyecto, incluyendo los peligros naturales que pudieran afectar su viabilidad (Reglamento SEIA - Anexo I). Como requisito para los Términos de Referencia del EIA semi-detallado (Reglamento SEIA Anexo III), se menciona, en el párrafo correspondiente a la Línea Base, que se debe incluir las unidades paisajísticas en la descripción del medio biológico. En el Anexo V del Reglamento SEIA se describen los criterios de protección ambiental (estrategia de protección), entre los cuales se menciona dentro del Criterio 4 (la protección de las áreas naturales protegidas) a la modificación de la composición del paisaje natural. Los estudios que requiere la norma peruana del EIA, no tienen reflejo directo y explícito en la organización del paisaje y los impactos no se reflejan generalmente en escenarios futuros de cambio, que representen el espacio, analicen los impactos y elijan alternativas favorables en el uso del suelo y su percepción tanto en el área de influencia directa como a escala regional. En el año 1969, cuando se establece en los EEUU el Environmental Act, el cual constituye la norma que crea el primer método para la Evaluación del Impacto Ambiental, el profesor Ian McHargh, propone un método para el análisis de la aptitud del paisaje en relación a posibles usos humanos; el método consiste en relacionar la estructura del paisaje con los procesos intrínsecos que lo forman y de dan un valor y lo categorizan en aquellos paisajes que son mas susceptibles a los impactos de naturaleza humana. La propuesta analítica de McHarg, ha sido continuado buscando la aplicación práctica de ideales teóricos; métodos operativos como el propuesto por el reglamento de Zonificación Ecológica Económica - ZEE (DS 087-2004), incluye métodos y criterios en el 
ideal de buscar espacios adecuados para determinados usos, pueden llegar a ser mucho más operativos y pasar de la etapa de evaluación hacia el modelamiento de alternativas futuras. Justamente la aplicación de los resultados obtenidos a partir del actual método de ZEE ha devenido en algunos casos en conflictos entre preservación y cambio, con implicancias y proyección que han alcanzado significancia en los dominios de la política. Al respecto Spirn (2000) citando a McHarg menciona la desesperada necesidad de profesionales que sean conservacionistas por instinto, pero que tengan no solo el interés por preservar, sino también por crear y gestionar.

\section{Resultados y discusión}

Pasemos ahora a exponer dos ejemplos de rápidos cambios que ocurren en el paisaje a escala regional: el primero es el fenómeno de urbanización en los valles agrícolas costeros de los ríos Chillón, Rimac y Lurín, creando el área metropolitana de Lima; y el segundo, en el desarrollo y variación del paisaje altoandino durante la fase de operación del complejo minero Yanacocha, ubicado en Cajamarca. El caso de la región metropolitana de Lima se caracteriza por ser un proceso de urbanización a gran escala, en un patrón generalizado globalmente como un fenómeno donde la población mundial tiende a localizarse cada vez mas en áreas urbanas, sobretodo en grandes conurbaciones. Particularmente en Lima este proceso sigue en operación y todavía viene ocupándose territorio originalmente agrícola, cambiando su uso a suelo urbano. El paisaje del área metropolitana de Lima abarca físicamente la superficie de tres valles costeros, los cuales de norte a sur son: Chillón, Rímac y Lurín.

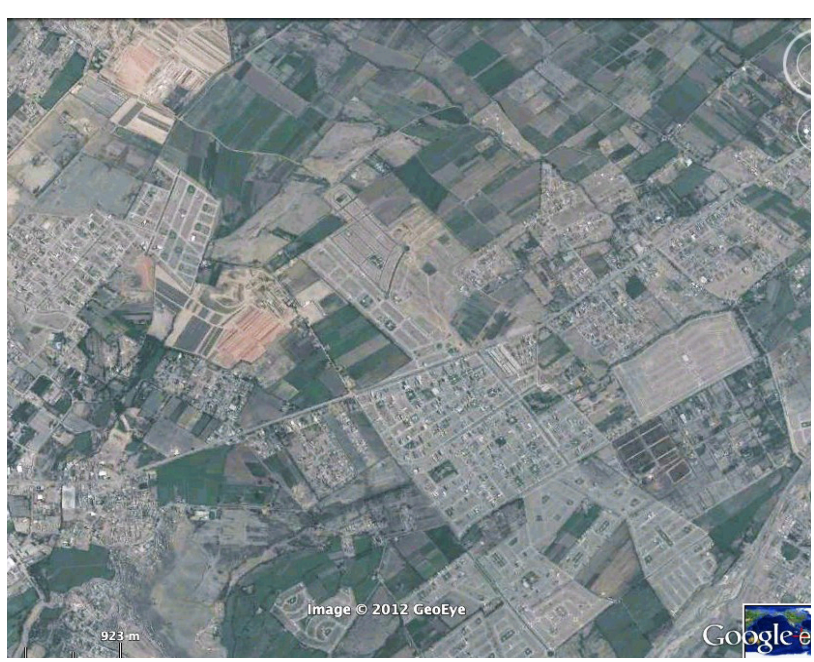

Figura 2. Urbanización sobre tierras agrícolas en el valle de Chillón (Fuente: Google Earth 2012).
El carácter original agrícola de los valles que conforman la región estaban compuestos mayoritariamente por una cobertura de parcelas e infraestructura de riego, la cual ha devenido en un espacio urbano mayoritario que ha cubierto, e impermeabilizado literalmente, el espacio e infraestructura agrícola original, sobretodo en el valle del rio Rímac; el desarrollo del denso tejido urbano ha sido limitado por las faldas y estribaciones del relieve montañoso tierra adentro. El motor del cambio fue la mayor rentabilidad que producía y todavía produce el valor de venta de terreno agrícola para uso urbano, en comparación con la renta que genera la producción agrícola en el corto plazo, así como el costo de oportunidad relacionado en las actuales condiciones del negocio inmobiliario, que actualmente evidencian un gran dinamismo. Las grandes extensiones agrícolas urbanizadas, en el marco de reglamentos de habilitación urbana muy generalistas, presentan un tejido urbano cuya densidad se viene incrementando.

Los espacios naturales remanentes del antiguo valle son los actuales parques, algunos aún son irrigados por los viejos canales de riego que todavía funcionan, como el famoso rio Surco. Este nuevo carácter del paisaje regional metropolitano tiene un patrón espacial particular al ser visto desde el aire; este es un entramado heterogéneo cuyo tejido está conformado por paños con diferentes patrones de hilado; esa metáfora se refiere a la existencia de diferentes modelos de urbanización y habilitación realizadas en forma independiente una de otra, por ejemplo comparemos las manzanas y calles de La Victoria con aquellas de San Borja o Villa el Salvador y veremos que el trazo, densidad y en general la configuración espacial de esos paños de tejido urbano varían entre si pero de alguna manera están unidos a través de las grandes vías o avenidas metropolitanas, las cuales si fueron planificadas a escala metropolitana. Un concepto sencillo que ayudara a entender y representar mejor este gran lienzo urbano; vamos utilizar una palabra llamada mosaico que el profesor y biólogo Richard T.T. Forman utilizó, en los años ochenta, para referirse al espacio regional visto desde el aire. Este paisaje observado a esa escala regional contiene un patrón particular de formas que cubren la superficie del suelo y que pueden simplificarse en tres elementos: fragmentos, corredores, y la matriz que es el lienzo de fondo que rodea a los dos primeros elementos. De acuerdo con Farina (2006) todo este conjunto de elementos materiales es el mosaico. Ya con anterioridad, en los años treinta, el geógrafo Carl Troll había utilizado fotografías aéreas en su investigación, a través de la cual intuyo que la forma o configuración espacial que presenta el mosaico estaba relacionada con los procesos y funciones ecológicas que se llevan a cabo dentro de su estructura, a este tipo de estudio Troll le dio el nombre de Ecología del Paisaje. 


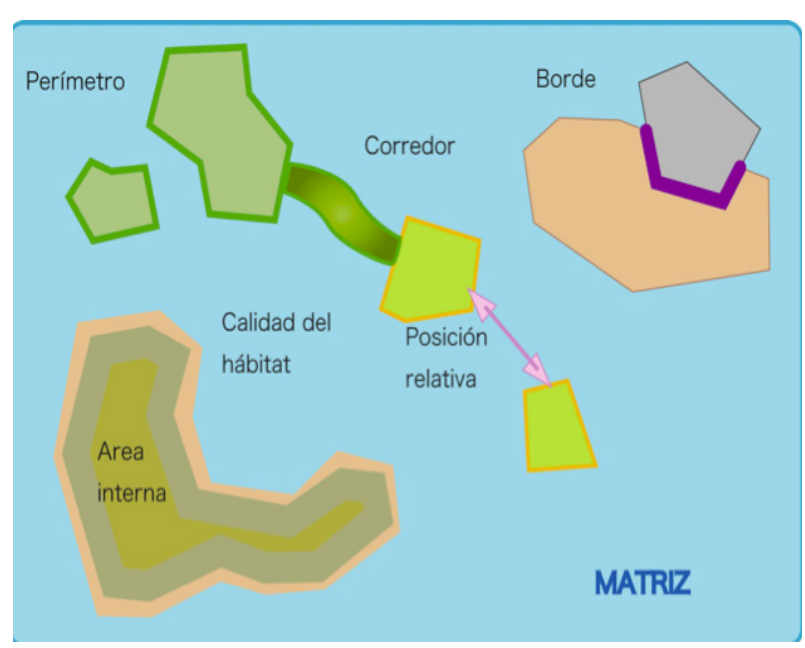

Figura 3. Modelo Matriz - Fragmento - Corredor.

Volviendo al caso de Lima, este mosaico tiene un patrón heterogéneo, donde esos paños contienen distintas densidades y formas, y su desarrollo inconexo, provocado quizá por el diseño y habilitación urbana aplicado individualmente por las distintas compañías urbanizadoras, cooperativas y movimientos informales. Esta práctica de diseño urbano propicio una pérdida de espacios naturales y un aislamiento de los pocos elementos naturales remanentes que en este caso son los parques urbanos. En vista de los resultados observados en el trazo y configuración espacial de los parques, no se planifico a escala metropolitana una malla de infraestructura verde, equivalente y equiparable a la red vial metropolitana, que dio como resultado los espacios verdes inconexos, incluso a escala distrital. Una palabra describe universalmente esta situación desde el punto de vista de la función ecológica del mosaico: fragmentación del paisaje natural. Según Collinge (1996), la perdida y aislamiento de los hábitats asociada a la conversión del territorio para actividades humanas, constituye la amenaza más seria a la diversidad biológica en el planeta. Jenerette y Wu (2001), señalan que, por ejemplo, convirtiendo zonas desérticas en áreas residenciales, se alteran muchos parámetros ambientales como las propiedades físicas y químicas del suelo, la disponibilidad de agua, la vegetación y las comunidades animales y microbianas. Este hecho serio de la fragmentación y perdida de la función natural en el paisaje, cuya evidencia es visible y cuyo análisis puede profundizarse, dependiendo de la escala de estudio y los objetivos que se consideren, adquiere importancia a partir del gran valor que últimamente la sociedad peruana viene dando tanto a la condición del medio ambiente, como al bienestar de los habitantes, y el mantenimiento de las funciones y servicios que la naturaleza provee: aire, agua, absorción de contaminantes, etc. Es por tanto importante solucionar el problema de la fragmentación, y en la medida de lo posible anticiparlo a través del planeamiento físico. En todo caso debe tenerse en cuenta que una vez fragmentado el paisaje, puede estimarse que su recuperación requiera enormes cantidades de dinero y un plazo de muchos años con el fin de obtener paisajes con una complejidad y sostenimiento mínimos, que luego permitan suponer el mantenimiento y evolución hacia un estado del paisaje con un mayor grado de maduración y complejidad que las condiciones del sistema territorial lo permitan. Al hablar de complejidad del sistema natural a construir, se entiende el establecimiento de un hábitat compuesto por especies de flora y fauna que se adapten a la degradación ocurrida en el suelo, agua y aire en los paisajes modificados por la actividad humana.

Para complementar al concepto del mosaico físico, incluyendo el componente perceptivo, el cual como mencionamos depende de los valores de la sociedad y además incluye el aspecto funcional de la recreación, citare como ejemplo el uso y usuarios de las áreas verdes públicas en la ciudad. En la región metropolitana de Lima el tejido social también es heterogéneo y no existe un solo modelo para el diseño, de las áreas verdes, que se adapte a las necesidades y usos recreativos de los diferentes grupos sociales. Un parque al ser utilizado por un determinado sector socio-económico es cuidado y mantenido de acuerdo a ciertos valores, que quizá otro sector socio-económico no los comparta o los sienta y considere extraños a sus propios valores y percepción. Este conflicto de valores, expresado por ejemplo en el uso y cuidado de un parque, genera en consecuencia una condición de incompatibilidad y poca identificación con ese parque. El estudio del paisaje en esos términos requiere de recursos financieros y tiempo para plasmar un sistema funcional adaptado a los diferentes usos y valores de la heterogénea sociedad limeña. Una aproximación sobre la heterogeneidad y el entendimiento de los usos y costumbres de los grupos sociales se puede observar en la localización y composición de la actual oferta inmobiliaria en la ciudad de Lima, cuyas superficies, distribución interna y acabados reflejan esa heterogeneidad social y cultural. De este ejemplo, los inmuebles más caros están ubicados en lo que se puede llamar "islas de bienestar" por contar con buenas condiciones de habitabilidad, servicios culturales y accesibilidad a espacios naturales de calidad. Que gran problema es entonces estudiar científicamente y crear espacios naturales aptos y útiles para la ciudadanía diversa, y al parecer ninguna disciplina profesional individual esta lo suficientemente entrenada para afrontar integralmente este desafío; el camino nuevamente se presenta largo en su plazo de tiempo. Es necesario un plan y la escala del área de intervención requiere de estrategias y políticas concordadas en los diferentes niveles de decisión: desde la gestión estratégica a escala metropolitana (publica y privada) hasta el proceso de diseño del parque vecinal (publico y privado). La demanda de espacios y recursos naturales que la sociedad peruana contemporánea exige, en la presente etapa de creciente incremento del consumo y acceso a bienes y servicios, destinados a mantener los también crecientes estándares de calidad de vida, se sustenta en gran medida en los ingresos económicos generados por la actividad minera a gran escala, cuyos productos son dirigidos 
principalmente a la exportación y son dependientes de los precios que rigen en el mercado global.

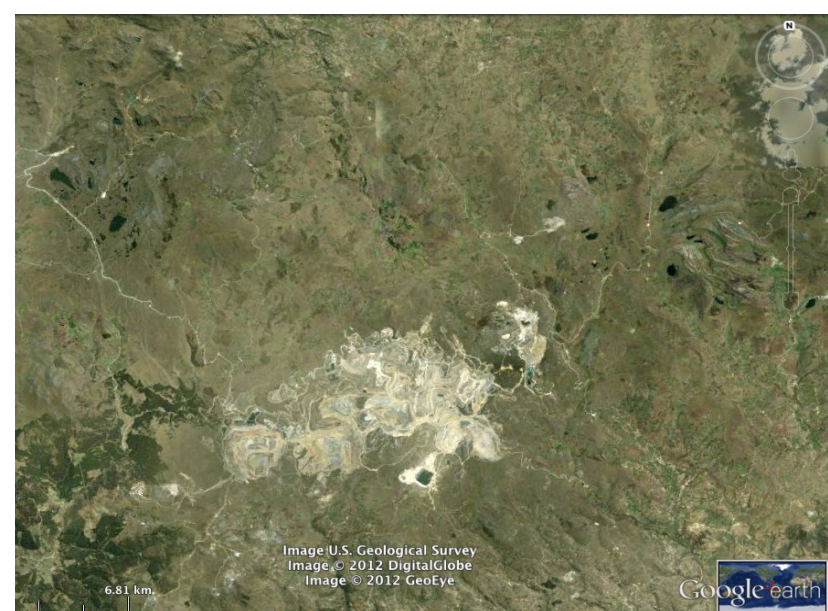

Figura 4. Mosaico territorial en la región altoandina mostrando el fragmento del área de operaciones del complejo minero Yanacocha (Fuente: Google Earth 2012).

Tal como señala Cherry (2008), los minerales y metales que han sido parte de la civilización humana desde sus orígenes, siempre han provenido de la tierra tal como ocurre actualmente; la diferencia está en la forma como los minerales y metales son extraídos, y también en los legados y oportunidades de la actividad minera en si misma. Según el mismo autor, las oportunidades sociales y económicas que se vienen aplicando, en algunos casos, vienen implementando materiales y tecnología del siglo XXI. La actividad de producción minera a gran escala y tajo abierto establecida en el Perú, dentro del marco de promoción de inversiones en vigencia desde los años noventa, tuvo entre sus primeras operaciones al complejo minero de Yanacocha, ubicado en Cajamarca. La operación minera que consta de varios sectores de explotación a tajo abierto, así como depósitos de desmonte y pilas de lixiviación, han modelado en su conjunto el relieve general del paisaje altoandino y su cobertura de pajonales y matorrales, dentro de los límites del área de operaciones. En un lapso de casi veinte años se ha creado una nueva estructura física, a través del movimiento de tierras, y han aparecido nuevos procesos de circulación de agua, nutrientes, y vida silvestre, resultando en un paisaje modelado de acuerdo a las necesidades y procedimientos de la extracción del mineral. Este nuevo paisaje, visto como un mosaico a escala regional, muestra al área de operaciones mineras como un gran fragmento de suelo alterado, rodeado por una matriz de cobertura vegetal altoandina de un carácter y uso del suelo rural, integrado en el relieve de las cumbres y la red hidrográfica.

Las condiciones naturales del paisaje en el complejo minero Yanacocha vienen siendo recuperadas a través de las actividades de cierre, en sectores donde han concluido las operaciones. En este punto es importante hacer una precisión referida al objetivo de las actividades de cierre; pues acertadamente estos trabajos no buscan la restauración de las condiciones originales, que serían difíciles de conseguir dados los recursos disponibles para volver a su estado original el paisaje; en este caso las actividades y el concepto puesto en práctica es el de recuperación a partir de las condiciones actuales de alteración, donde por ejemplo la cobertura vegetal, la pendiente y la acidez del suelo han sido afectados negativamente de modo que solo es posible la supervivencia de comunidades vivas que resistan entornos muy agresivos. Es en estas adversas condiciones que la actividad de recuperación del paisaje tiene el desafío científico y creativo para aceptar el hecho de la alteración evaluándola objetivamente y buscar alternativas de uso del suelo que no contrasten con el entorno rural y el carácter general del paisaje en la región. Los estudios de evaluación de impacto ambiental y los planes del cierre de minas proponen medidas de mitigación, basadas en indicadores de calidad y performance ambiental de los recursos abióticos y bióticos presentes antes del inicio de las operaciones, estos indicadores son monitoreados continuamente. Del mismo modo los estudios de línea base social describen con claridad la estructura, perfil socioeconómico y los valores que gobernaban el comportamiento de las poblaciones y organizaciones públicas y privadas de la zona, las cuales se han transformado a la misma velocidad con la que el paisaje ha mutado. Los procesos son conocidos y vienen siendo registrados detalladamente, a pesar de ello se aprecia que la correlación entre las variables bióticas, abióticas y culturales y su integración en un sistema territorial amplio todavía no ha sido documentado ni implementado, a nivel de estrategias y políticas que agrupen, en un solo paquete tecnológico interrelacionado, los ámbitos de intervención públicos y privados, en la escala regional, los cuales en la actualidad están en conflicto. El concepto más holístico del paisaje como espacio cuyo carácter es el resultado de la acción e interacción de procesos naturales y humanos funciona en el área del complejo minero Yanacocha, pero en vista del descontento social que en este caso se manifiesta en la percepción negativa que se tiene del paisaje resultante, se puede entender que se requiera un estudio integral que justifique y relacione en un plan del paisaje mas amplio, que informe las decisiones tomadas respecto a las actividades y programas de recuperación del paisaje y su influencia en el desarrollo rural, tanto en las zonas de cierre como en los anillos de influencia externos al área de operaciones.

\section{Conclusiones}

Los cambios de uso del suelo descritos son desde nuestra posición inevitables en vista de la actual demanda de bienes y servicios que la sociedad viene requiriendo y cuya proyección futura indica que se va incrementando esa demanda. En los casos descritos la demanda es por espacio físico y recursos minerales, ambos destinados 
a usos de la sociedad. El cambio es por tanto inevitable y el carácter original de los paisajes afectados tienden a presentar nuevos componentes tanto en su estructura como en los procesos que se relacionan con ella. Asimismo se ha mencionado que el paisaje cambia no solo por intervención humana sino por fuerzas propias de la naturaleza las cuales se ocurren a partir de condiciones climáticas y geológicas en un momento dado el cual es irrepetible. Esto último lleva a establecer que la restauración de las condiciones originales, como manifiesta Del Tredici (2008), es casi imposible pues primero tendrían que volverse a reconstruir el relieve y la cobertura así como los procesos naturales relacionados, y además tendría que repetirse o recrearse las condiciones climáticas originales del momento y las condiciones elegidas de restauración. Por esa razón es que se considera que la palabra y concepto de recuperación es el más indicado a usar en los casos de procesos de mutación en el uso suelo tan acelerado como la urbanización o la operación minera a gran escala.

Los dos ejemplos elegidos para describir los procesos de cambio permanente en el paisaje urbano y rural, han sido escogidos por su relevancia referida tanto a la gran escala o superficie que abarca, como a la rapidez con que el cambio se ha producido. Se ha sugerido asimismo que el motor que dirige el cambio responde a condiciones sociales, técnicas y al marco legal de una economía de libre mercado y podrían resultar ejemplos paradigmáticos de cómo esas condiciones influyen en el carácter del paisaje. En todo caso lo que si podríamos convenir es que la percepción social del paisaje resultante es cada vez más crítico respecto al resultado, por lo menos visual, de la alteración muestra, en ambos ejemplos. Algo que todavía resulta invisible para mucha gente, con excepción de los técnicos y estudiosos de la geografía y los procesos naturales, es que los procesos subyacentes y relacionados a la alteración de la estructura visible del paisaje, han sido alterados de un modo que su restauración es inviable económicamente, socialmente y ambientalmente. Así como ha cambiado el paisaje, los modos de vida y valores sociales también han cambiado. Descrito el problema, su complejidad e importancia procederemos a sugerir una solución positivista de la mano de dos actividades secuenciales y complementarias: la evaluación y el planeamiento del paisaje, con un ingrediente añadido que es el enfoque transdisciplinario. La evaluación consiste en responder la pregunta de ¿cómo está funcionando el paisaje? Y el planeamiento se refiere a responder otra pregunta ¿de qué manera debe ser modificado el paisaje? Ambas preguntas y secuencia lógica forman parte de la estructura que propone Carl Steinitz (1997), para el planeamiento sostenible del paisaje. El ingrediente transdisciplinario se refiere a que tanto en la evaluación como en el plan se requiere la intervención por igual de los profesionales como de la población y grupos de interés. Aunque no hay mayor experiencia práctica en estudios de carácter transdisciplinario, su utilidad es reconocida con mayor frecuencia en la literatura científica. El rol de la población y los grupos de interés en el planeamiento e implementación de las actividades en el paisaje abarca un rango desde solo ser informado, consultado, participar en el plan y controlarlo (Tress y Tress 2005). Se requieren tiempo y recursos económicos para implementar y hacer operativo estos requerimientos metodológicos.

Los estudios que exige actualmente la normatividad publica, tales como EIA, ZEE, Planes de Desarrollo Urbano, Planes de Cierre de Minas, etc. son intervenciones que en la práctica recuperan el paisaje notablemente respecto a sus condiciones previas, es de esperar que en un horizonte temporal de largo plazo se aprecie mejor su influencia regional; ejemplos de recuperación del paisaje como la revegetación del tajo Maqui Maqui en Yanacocha, o la renovación del equipamiento urbano en la playa La Herradura, en la Costa Verde, esperan ser estudiados en mayor detalle, y no solamente estudiados sino también evaluados y planificados pues estas dos actividades son cíclicas y deben ser realizadas en forma constante porque las condiciones del entorno ambiental, social y económico nunca permanecen estáticas. El modo de estudio del paisaje regional, requiere reconocer que no existe en el Perú una particular disciplina profesional oriente la labor de creación y planeamiento de los espacios abiertos desde la escala del jardín al tamaño de región; la falta de una línea de investigación y estudio académico institucionalizado en una carrera profesional, como la arquitectura del paisaje, no es condición imprescindible para el estudio del paisaje, puesto que hay otras profesiones que estudian y sintetizan los conocimientos del clima, la hidrología, los suelos, la geología, la vegetación, la organización del espacio, etc. el problema que se observa es la falta de un enfoque integrador o interdisciplinario, que fuerce un cambio en los actuales usos y costumbres multidisciplinarios, donde por ejemplo los estudios de línea base ambiental y social tienen múltiples objetivos (dependiendo de cada disciplina), que no se entrecruzan para crear nuevos conocimientos.

El desafío o respuesta preliminar, y provisional dado el seguro avance y actualización en el estudio del paisaje regional peruano, y la integración profesional disciplinaria, está inspirado en el esquema metodológico propuesto por Steinitz. La respuesta planteado en términos de realizar las siguientes actividades: estudio del carácter presente del paisaje, entendimiento de una determinada trayectoria de cambio, y la comprensión de los procesos que rigen ese cambio; el conocimiento así generado permitirá realizar la evaluación y proponer un plan para adaptar el paisaje a las inevitables e impredecibles condiciones futuras, en la búsqueda del paradigma de la sostenibilidad y sus resultados tangibles como los servicios ambientales y el bienestar que provee a la sociedad. Finalmente se sugiere estudiar el paisaje en la escala regional porque provee una visión integral de las actividades naturales y humanas; la mayor amplitud espacial permite observar las singularidades y elementos físicos del territorio, desde una perspectiva amplia, permitiendo un análisis del conjunto y no solo de cada parte del territorio. El 
enfoque totalizador del paisaje ayuda, por ejemplo, a descubrir un sistema de relaciones espaciales, entre los elementos abióticos bióticos y culturales, dentro de áreas intervenidas por el hombre, donde la naturaleza aún persiste, y donde se relacionan los diferentes impactos (positivos y negativos) en una forma que se configura espacialmente en una estructura cuantificable, cuya percepción le confiere un carácter particular, resultando en un paisaje posible de ser evaluado y planificado, en condiciones de cambio a escala regional.

\section{Literatura citada}

Antrop, M. 2005. From holistic landscape syntesis to transdisciplinary landscape management. In: Tress, B; Tress, G; Fry, G; Opdam P. eds. From Landscape Research to Landscape Planning: Aspects of Integration, Education and Application. p. 13-26.

Cherry, J. 2008. Case studies of successful reclamation and sustainable development at Kennecott mining sites. En: A. M. Berger (2008), Designing the Reclaimed Landscapes 105 - 112. Taylor y Francis, New York, USA.

Collinge, S. K. 1996. Ecological consequences of habitat fragmentation: implications for landscape architecture and planning. Landscape and Urban Planning 36: 59-77.

Jenerette, G. D. and Wu, J. 2001. Analysis and simulation of land-use change in the central ArizonaPhoenix región, USA. Landscape Ecology 16: 611-626.

Spirn, A. W. 2000. Ian McHarg Landscape Architecture and Environtalism: Ideas and Methods in Context. In: Conan M. ed. Environtalism in Landscape Architecture, 97-114. Dumbarton Oaks Research and Library Collection, Washington DC, USA.

Steinitz, C. 1997. An Alternative Future for the Region of Camp Pendleton, California. Harvard University, Graduate School of Design. Documento de Trabajo.

Tress, G.; Tress, B. and Fry, G. 2004. Clarifying integrative research concepts in landscape ecology. Landscape Ecology 20: 479-493. 